\title{
Ärztliche Weiter- und Fortbildung unter Corona
}

\author{
Dina-Maria Jakob ${ }^{a}$, Patrizia Kündig ${ }^{a}$, Reto Thomasina, Simon Stettlerb, Monika Brodmann Maeder ${ }^{\mathrm{c}}$ \\ a Dr. med., Verband Schweizerischer Assistenz- und Oberärztinnen und -ärzte, vsao; ${ }^{b}$ Geschäftsführer vsao; \\ c PD Dr. med., Präsidentin Schweizerisches Institut für ärztliche Weiter- und Fortbildung SIWF
}

Das SIWF hat während der Pandemie nicht nur allen fortbildungspflichtigen Ärztinnen und Ärzten Credits für 2020 und 2021 geschenkt, sondern versuchte auch, mit unbürokratischen Massnahmen Druck von Weiterzubildenden, Weiterbildungsstätten und Fachgesellschaften zu nehmen [1]. Um das Ausmass der Pandemiefolgen auf die ärztliche Weiterbildung zu beleuchten, wird die jährliche Befragung zur Qualität das Thema «Weiterbildung während der Pandemie» aufnehmen. Mit diesem Beitrag aus den Reihen des vsao kommen direkt Betroffene zu Wort.

\section{Umwälzungen im Spitalbetrieb durch Corona}

Die Corona-Pandemie traf das Schweizer Gesundheitswesen im März 2020 mit grosser Wucht und wenig Vorlaufzeit. Vieles wurde von einem Tag auf den anderen auf den Kopf gestellt, so auch die ärztliche Weiter- und Fortbildung. Im ersten Moment ging es für die Spitäler, für das Personal und für die Patientinnen und Patienten um das Überleben - und das im wahrsten Sinn des Wortes. Während ein Teil der Kliniken und Spitalabteilungen auf Geheiss des Bundesrats geschlossen wurden und sie ihr Personal nach Hause schicken mussten, wurden andere zu Corona-Stationen umgebaut oder das Personal auf andere Stationen umverteilt. Intensivund Notfallstationen konnten die Arbeitslast bei der Be-

Nach über einem Jahr Pandemie muss diese als eine Art «neue Normalität» akzeptiert werden.

handlung von COVID-19-Patientinnen und -Patienten kaum bewältigen. Klar, dass in dieser Zeit auch die ärztliche Weiter- und Fortbildung hintenanstehen musste. Nach der ersten hektischen Phase liefen die Spitäler über mehrere Monate in einer Art «Corona-Betrieb». An die Durchführung ärztlicher Weiter- und Fortbildung war in dieser Zeit kaum zu denken. Das SIWF bemühte sich nach Kräften und unter Einbezug aller Beteiligten, mit Spezialregeln der ausserordentlichen Situation der Assistenz- und Oberärztinnen und -ärzte gerecht zu werden (www.siwf.ch $\rightarrow$ Themen $\rightarrow$ Covid-19).

\section{Einschränkungen der Weiterbildung}

Im Frühsommer durften die geschlossenen Abteilungen ihre Arbeit wiederaufnehmen. Leider ignorierte die Politik in dieser Zeit die wiederholt geäusserten Sorgen der Spitäler wegen der finanziellen Auswirkungen der Pandemie. Die Konsequenzen waren absehbar. Einige Abteilungen steckten weiterhin im CoronaStress fest, andere mussten neben dem wiederaufgenommenen Normalbetrieb möglichst viele aufgeschobene Behandlungen nachholen. Dabei wurde die wiedergewonnene Handlungsfreiheit in den Kliniken nicht überall genutzt, um auch die Weiterbildung wieder hochzufahren. Dem vsao sind Beispiele bekannt, wo an Samstagen in Kliniken Wahleingriffe nachgeholt wurden, ohne dass dazu mehr Personal zur Verfügung gestanden hätte. Bei solchem Arbeitsdruck wird Weiter- und Fortbildung kaum Platz eingeräumt. Wie so oft waren die Prioritäten klar wirtschaftlich orientiert und zuungunsten der Weiter- und Fortbildung.

Es war allen Beteiligten bewusst, dass wegen der Corona-Pandemie die ärztliche Weiter- und Fortbildung zeitweise massiv eingeschränkt werden musste. Sie ist aber kein nice to have, sondern eine rechtliche Pflicht. Die Weiterbildungsstätten sind verpflichtet, ihren Assistenzärztinnen und -ärzten die versprochene Weiterbildung zu bieten. Die Assistenz- und Oberärztinnen und -ärzte wiederum sind verpflichtet, ihre Weiter- und Fortbildung zu absolvieren. Das heisst, «sobald die Pandemie das zulässt», müssen auch Weiter- und Fortbildungen wieder durchgeführt werden. 


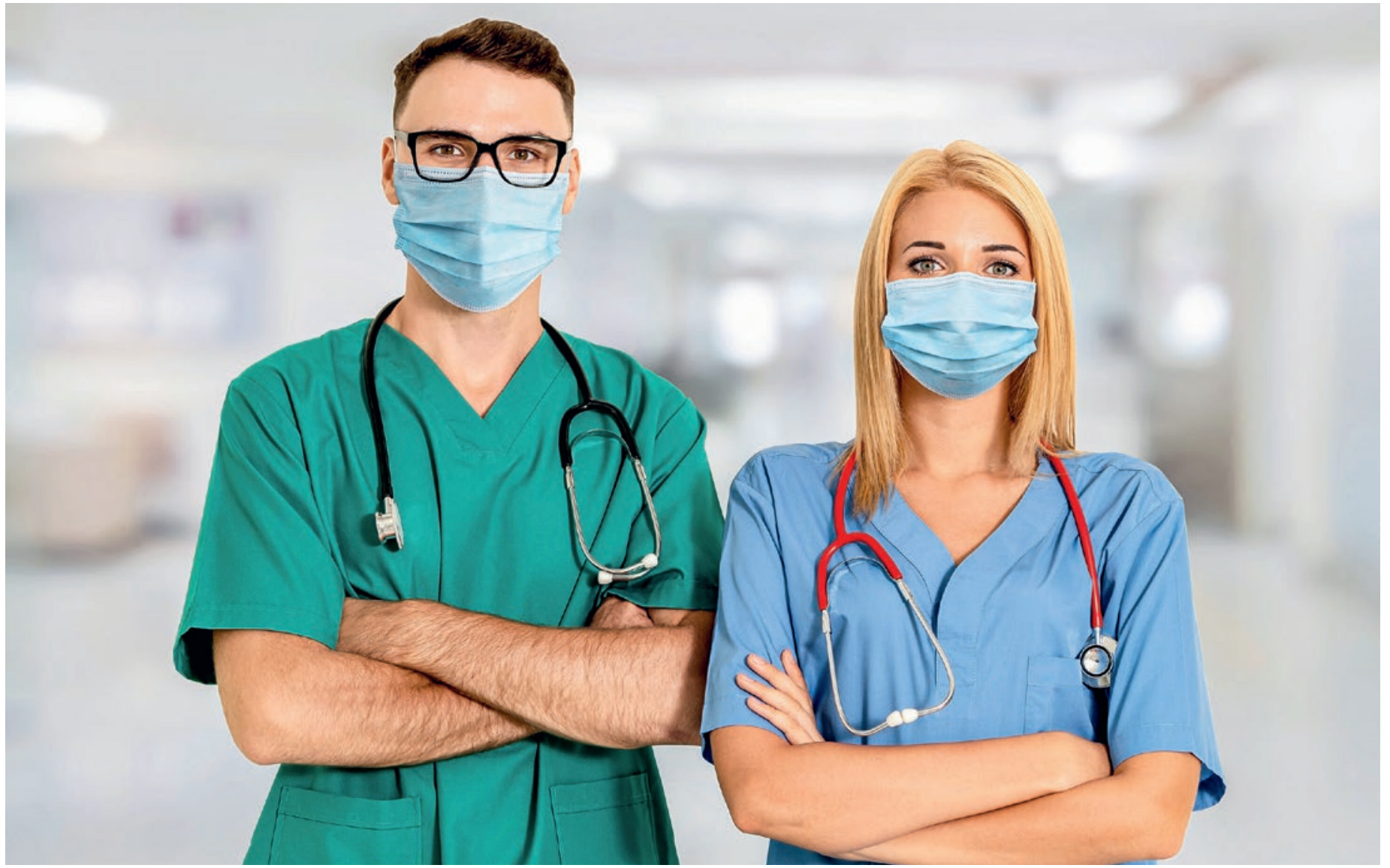

Auch in Zeiten der Pandemie haben Assistenz- sowie Oberärztinnen und -ärzte ein Anrecht auf Weiterbildung.

Es gab keinen fixen oder gar offiziellen Termin, an dem Weiter- und Fortbildung wieder starten konnten bzw. mussten. Die Bedingungen in den einzelnen Kliniken und Spitälern waren zu unterschiedlich. Der vsao kennt Beispiele, bei denen sehr schnell Wege gesucht und gefunden wurden, um Weiterbildung zu ermöglichen. Ebenso haben wir von Fällen gehört, in denen die Pandemie als Entschuldigung vorgeschoben wurde, um die Weiterbildung möglichst lange zu vermeiden.

\section{Möglichkeiten zur Aufrechterhaltung von Weiter- und Fortbildung}

Nach über einem Jahr Pandemie muss diese aber als eine Art «neue Normalität» akzeptiert werden. Corona ist immer noch da und wird uns auch weiterhin begleiten. Die Spitäler und das Personal haben sich so gut wie möglich darauf eingestellt, und die Weiterbildung (und Fortbildung) muss zwingend wieder durchgeführt werden. Mittlerweile scheint es denn auch mehr eine Frage der Motivation, Innovation und Offenheit der Weiterbildenden und der Weiterbildungsstättenleiterinnen und -leiter zu sein. Videokonferenzen sind zu einem festen Bestandteil im Berufsalltag und in der Aus-, Weiter- und Fortbildung quer durch alle Berufe und sogar der (Primar-)Schulen - geworden. Es gibt genügend gute Beispiele, wie ärztliche Weiterbildung via Videoübertragung, manchmal sogar inklusive prakti- scher Live-Beispiele oder Fallbesprechungen, durchgeführt werden kann. Teilweise werden Bildungsveranstaltungen und Vorträge aufgezeichnet, wodurch die Ärztinnen und Ärzte die Videos auch später noch als Podcast herunterladen und anschauen können. Dies

Es gibt genügend gute Beispiele, wie ärztliche Weiterbildung via Videoübertragung durchgeführt werden kann.

bietet nicht nur den in Teilzeit und im Schichtbetrieb arbeitenden Ärztinnen und Ärzten die Möglichkeit, sich die Videos jederzeit anzusehen. Es sollte auch als Chance genutzt werden, um eine Art Bibliothek mit den verschiedenen Vorlesungen und Vorträgen anzulegen und diese möglichst übergreifend in einem Spitalnetz kantonal oder sogar schweizweit einzusetzen. Weiterbildungen und Workshops können in kleinen Gruppen mit Abstand, eventuell hybrid mit Zuschaltung eines Teils der Teilnehmenden via Videoübertragung abgehalten werden. Im Sinne von blended learning können Selbststudium und (virtuelle) Fragestunden kombiniert werden. Die Aufzählung ist sicher nicht abschliessend.

\section{Gerne wieder ohne Videoübertragung}

Um Missverständnisse zu vermeiden: Es geht hier nicht darum, Weiterbildung via Videostreaming oder 
Podcast als Allerweltsheilmittel darzustellen. Wir alle wünschen uns die direkte Diskussion in der Gruppe oder das praktische Teaching vor Ort zurück. Einerseits erfordert die aktuelle Situation aber das Ausweichen auf Alternativen, und andererseits haben die vergangenen Monate - dort, wo die Alternativen gesucht und genutzt wurden - durchaus auch Potenzial gezeigt, die ärztliche Weiter- und Fortbildung weiterzuentwickeln und beispielsweise die Beteiligten besser zu vernetzen. Wenn Assistenzärztinnen und -ärzte in kleineren Spitälern auf diese Weise vermehrt von Weiterbildungsangeboten in grösseren Spitälern profitieren können, ist das ein Gewinn. Wenn Ärztinnen und Ärzte wegen Schicht- oder Teilzeitarbeit eine Weiterbildung nachträglich anschauen können, ist das ein Gewinn. Wenn jemand an einer Lehrveranstaltung teilnehmen kann, weil sie/er sich die Reisezeit erspart, ist das ein Gewinn.

\section{Neustart erforderlich - Weiter- und Fortbildung ist Recht und Pflicht}

In verschiedenen Kliniken und Spitälern ist es den Verantwortlichen (und dazu gehören die Weiterbildner ebenso wie die Weiterzubildenden) gelungen, mit viel Einsatz und Motivation die Weiterbildungen wieder hochzufahren. Es gibt aber leider immer noch zu viele Weiterbildungsstätten, die nichts davon umsetzen. Eine Umfrage der vsao-Sektion Bern hat einerseits das grosse Ausmass der wegen Corona weggefallenen Weiterbildung bestätigt. Andererseits hat sie aber auch gezeigt, dass die Weiterbildung bei weitem noch nicht überall wieder angeboten wird. Knapp 40\% der Teilnehmenden gaben an, dass bei ihnen ausgefallene Weiterbildungen gar nicht durch virtuelle Weiterbil- dungen ersetzt worden sind, und bei fast ebenso vielen war dies nur teilweise der Fall. Die Umfrage fand im Januar und Februar dieses Jahres statt. Nach über einem Jahr der Pandemie droht die Qualität der ärztlichen Weiter- und Fortbildung nachhaltig zu leiden.

Knapp 40\% der Teilnehmenden gaben an, dass ausgefallene Weiterbildungen nicht durch virtuelle Weiterbildungen ersetzt worden sind.

Eine gute Weiterbildung ist essentiell, und sie steht den Assistenzärztinnen und -ärzten auch zu. Diese können und sollen deshalb ihr Recht auf Weiterbildung einfordern, dabei aber auch ihren Teil dazu beitragen. Das Gleiche gilt für die ärztliche Fortbildung. Die geforderten Credits sind das eine, es geht aber primär um den Erhalt und die Erweiterung der ärztlichen Kompetenzen und damit um Qualitätssicherung. Die Diskussionen im Geschäftsausschuss des vsao und mit den Vertreterinnen und Vertretern der vsao-Sektionen zeigen für die ganze Schweiz ein ähnliches Bild. Es ist wie so oft, es braucht einen Willen, Interesse und Engagement, dann ist vieles möglich. Wer selber keine passende Lösung sieht, findet im Austausch mit anderen Kliniken viele gute Beispiele dafür, was alles möglich ist und funktioniert. Weiter- und Fortbildung sind Pflicht und eine grundlegende Voraussetzung für ein qualitativ hochstehendes Gesundheitssystem.

\section{Bildnachweis}

BiancoBlue | Dreamstime.com

\section{Literatur}

1 Hänggeli C. Notrecht in der Weiterbildung? Wie das SIWF auf die Herausforderungen von Corona reagierte. Schweiz Ärzteztg. 2020;101(31-32):924. https://doi.org/10.4414/saez.2020.19104 